\title{
Effects of Zoledronic Acid Treatment on Fracture Healing, Morbidity and Mortality in Elderly Patients with Osteoporotic Hip Fractures
}

\author{
Serdar Sargin ${ }^{1}$, Mehmet N Konya ${ }^{2}$, Anıl Gulcu ${ }^{3}$, Ahmet Aslan ${ }^{4}$
}

\begin{abstract}
Background: In this study, the effects of zoledronic acid (ZolA) administered at different times to patients undergoing surgical treatment for hip fracture were investigated.

Materials and methods: Ninety patients who underwent surgical treatment for osteoporotic (OP) hip fractures between February 2013 and September 2016 in our hospital were included in the study. After surgical treatment, patients were allocated into three groups: group I-patients who had osteosynthesis using proximal femoral nail (PFN) for an intertrochanteric fracture of the femur were given ZolA within 1 week after fracture and before discharge; group II-patients who had osteosynthesis using the PFN for an intertrochanteric fracture of the femur were given ZolA within 1 month after fracture post-discharge; group III-patients in the same age group who had a hemiarthroplasty (HA) for an intertrochanteric fracture of the femur were administered ZolA before discharge. In addition, all patients were given daily oral calcium and vitamin D3. The Radiographic Union Score for Hip (RUSH), Harris Hip Score (HHS), and bone mineral density (BMD) were used at the follow-up as evaluation criteria, and complications were noted.

Results: There were no significant differences between groups in terms of demographic data and laboratory outcomes $(p>0.05)$. Radiographic Union Score for Hip scores were similar between groups I and II $(p>0.05)$. Fracture union occurred by the sixth month in all patients whose results were evaluated. No statistically significant difference was found between three groups $(p>0.05)$. There was no difference between the three groups in the hip and vertebrae BMD and $t$ scores $(p>0.05)$. When $t$ and BMD scores before treatment were compared with those at 1 year after treatment, a benefit from ZolA treatment was observed in all three groups $(p<0.05)$.

Conclusion: This study shows that the timing of ZolA administration has no effect on fracture healing and complication incidence in elderly patients with hip fractures. In addition, ZolA was found to be beneficial in increasing BMD of both femur and vertebra in all groups, but there was no significant difference between the groups.

Clinical significance: The study demonstrated that ZolA may be used early in the treatment of osteoporotic hip fractures with PFN.

Keywords: Fracture healing, Harris Hip Score, Osteoporotic hip fracture, RUSH score, Zoledronic acid.

Strategies in Trauma and Limb Reconstruction (2019): 10.5005/jp-journals-10080-1439
\end{abstract}

\section{INTRODUCTION}

Hip fractures are the most important cause of morbidity and mortality in osteoporotic (OP) elderly patients. Osteoporotic hip fractures increase with age globally. ${ }^{1-3}$ Prevention and treatment of osteoporosis may prevent hip fractures and their co-morbidities. Bisphosphonates (BPs) are drugs whose efficacy in decreasing the risk of OP hip fracture has been proven. ${ }^{4}$ Zoledronic acid is a potent $\mathrm{BP}$ used in the treatment of osteoporosis and has been shown to reduce the risk of OP hip fractures significantly. ${ }^{5-7}$ Bisphosphonate treatment inhibits osteoclast-mediated bone resorption in order to prevent bone loss and to increase the strength of bone. ${ }^{6,8,9}$ However, osteoclasts are important in remodelling callus formation into lamellar bone. Therefore, concerns remain about the influence of BPs on the fracture healing process. ${ }^{8}$ In fracture healing, osteoclastic activity peaks within the first 2 weeks. The initial callus formation is completed in 3 weeks and then begins to calcify. At about 1 month after fracture, calcified cartilage and woven bone is formed within the callus mass. ${ }^{10,11}$ Bisphosphonate treatment, which inhibits osteoclastic activity and has an antiresorptive effect, is influential in this process.

Although BP treatment is known to reduce secondary fracture and mortality rates after hip fractures, the optimal timing of BP administration in elderly patients with hip fractures remains
${ }^{1}$ Department of Orthopaedics and Traumatology, Balıkesir University, Balıkesir, Turkey

${ }^{2}$ Department of Orthopaedics and Traumatology, Afyon Kocatepe University, Afyonkarahisar, Turkey

3,4 Department of Orthopaedics and Traumatology, Alaaddin Keykubat University, Alanya, Antalya, Turkey

Corresponding Author: Serdar Sargin, Department of Orthopaedics and Traumatology, Balıkesir University, Balıkesir, Turkey, Phone: +90 5073041370, e-mail: drserdarsargin@yahoo.com

How to cite this article: Sargin S, Konya MN, Gulcu A, et al. Effects of Zoledronic Acid Treatment on Fracture Healing, Morbidity and Mortality in Elderly Patients with Osteoporotic Hip Fractures. Strategies Trauma Limb Reconstr 2019;14(3):126-131.

Source of support: Nil

Conflict of interest: None

unclear. $^{12}$ In patients with ongoing fracture healing, intravenous BP administration has several effects. These include effects on bone mineral density (BMD), prevention of secondary fracture development, effects on survival, and effects on fracture healing. ${ }^{5,9}$ The study question, in the light of this information, is "What should be the optimal timing of biphosphonate treatment in order to benefit from its positive effects and avoid negative effects?" 
The aim of this study was to investigate the effect of zoledronic acid (ZolA) on mortality, morbidity, and fracture healing in patients who underwent surgical treatment for hip fractures.

\section{Materials and Methods}

In this prospective controlled study, patients who underwent surgery for OP hip fractures between February 2013 and September 2016 in Afyonkarahisar State Hospital were considered. Seventythree patients met the inclusion criteria out of the 90 enrolled and were included in the analysis (Table 1). After surgical treatment, patients were allocated into three groups:

Group I-patients who had osteosynthesis using proximal femoral nail (PFN) for an intertrochanteric fracture of the femur were given ZolA within 1 week after fracture and before discharge.

Group II-patients who had osteosynthesis using the PFN for an intertrochanteric fracture of the femur were given ZolA within 1 month after fracture post discharge.

Group III-patients in the same age group who had an HA for an intertrochanteric fracture of the femur were administered ZolA before discharge. This group was created to evaluate the effectiveness of ZolA. A placebo group was not used in this study due to ethical reasons. All patients were given a loading dose 50,000 IU of vitamin D3 (oral) followed with daily oral supplementation of $800 \mathrm{IU}$ of vitamin D and 1,200 mg elemental calcium.

\section{Classification of Fractures and Treatment}

Patients with extracapsular intertrochanteric Arbeitsgemeinschaft für Osteosynthesefragen (AO) type 31A1 and $A 2$ fractures underwent osteosynthesis with a PFN, whereas those with intracapsular $A O$ type 31B2 and B3 fractures had an HA using an endoprosthesis. Both were included in the present study.

\section{Randomisation}

No randomisation was made for surgical treatment. Osteosynthesis with the PFN was carried out depended on the type of fracture,

Table 1: Inclusion and exclusion criteria

\begin{tabular}{ll}
\hline Inclusion criteria & Exclusion criteria \\
\hline $\begin{array}{l}\text { Fractures associated with } \\
\text { osteoporotic low-energy } \\
\text { trauma, }\end{array}$ & $\cdot$ BP intolerance history \\
- In DEXA screening carried \\
$\begin{array}{l}\text { out previously and/or during } \\
\text { zoledronate treatment } \\
\text { according to WHO criteria } \\
\text { t score of }<-2.5,\end{array}$
\end{tabular}

patient factors, and preference of surgeon. However, ZolA administration was randomised.

\section{Sample Size}

Based upon previous studies ${ }^{8,12}$ for three groups, a variance analysis was performed, and with a large effect size of 0.4 , an $\alpha$ error value of 0.05 , and a power of 0.80 (power study for a significant $p$ value when the probable lowest degree of freedom as 3 and probable largest value of $f$ was calculated as 2.74), the required overall sample size was found to be 73 . Due to concern about probable deaths and loss of patients during follow-up, we had planned to include 30 patients in each group.

\section{Evaluation of Delay in Bone Union}

Patients were diagnosed with a delay in bone union at the end of the third month (mean: with a minimum of at least 6 weeks postsurgery) by the presence of at least one of the following radiological criteria:

- Lack of a change in the fracture line appearance when compared with the first postsurgical radiograph

- Detection of a fracture line present at the base with computed tomography or magnetic resonance image

- Displacement of the fracture despite fixation

- Callus formation in bone cortex but lacking evidence of consolidation, and

- Clinically in the presence of at least one of the following:

- Pain,

- Difficulty in walking, or

- Not being able to walk at all. ${ }^{9}$

\section{Evaluation of Bone Union}

Patients were invited at 3 and 6 weeks and again at 3, 6, and 12 months post-surgery for a radiological and clinical review. At these visits, anteroposterior and lateral hip radiography was obtained and bone healing assessed using a Radiographic Union Score for Hip (RUSH). ${ }^{13,14}$ Radiographic Union Score for Hip is a scoring system evaluating four components in radiography: cortical bridge index score, 4-12 points; cortical disappearance (of fracture line) score, 4-12 points; and two trabecular indices, one for consolidation and the other relating to the disappearance of the fracture line, 1-3 points. The overall RUSH score is between 10 and $30 .^{13,14}$

\section{Evaluation of Functional Results}

The clinical outcome was evaluated with the Harris hip score $(\mathrm{HHS})^{15,16}$ at the 12th month postoperatively. Scores are calculated as follows from a maximum of 100 (best possible result): pain (1 item, 0-44 points), function ( 7 items, $0-47$ points), absence of deformity ( 1 item, 4 points), and range of motion ( 2 items, 5 points). If the overall score is $<70$, it is considered as poor, whereas scores between 70 and 80 are moderate outcomes, those between 80 and 90 as good, and scores $>90$ as excellent. 15,16

\section{Evaluation of BMD}

Patients were screened for their BMD before treatment and on the 12th month postoperatively. In the anterior-posterior direction, vertebra (L1-L4) and femur upper end (totalhealthy side) measurements were made in accordance with recommendations. ${ }^{1,4,17}$ The screening was carried out with a dualenergy X-ray absorptiometer (DEXA). Patients with osteoporosis 
were detected according to their $t$ scores using World Health Organization (WHO) criteria. Care was taken for maintenance and calibration of the device and for all DEXA measurements to be made in the same hospital and by the same technician.

\section{Evaluation of Adverse Effects}

General adverse effects have been known to be flu-like symptoms, headache, joint and muscle pain, nausea, and vomiting with severe adverse effects to be cardiovascular, renal, and neurovascular effects and death. ${ }^{7}$

\section{Other Adverse Effects}

In addition, in order to rule out other causes of pain, lumbar anteriorposterior and lateral radiographs were taken to rule out any OP lumbar vertebral fracture.

\section{Follow-up}

All patients were monitored until certain end-points were reached. This included revision surgery if deemed necessary: a fracture in the other hip, death, or for 1 year after the index surgery. In the planned periodic follow-up visits, functional status and bone healing were recorded. At the end of the follow-up year, the bone healing period (groups I and II), adverse effects of ZolA, and other postoperative complications such as nonunion, infection, etc. were recorded, and the BMD values were measured.

\section{Statistical Analysis}

SPSS 20 version (SPSS, Inc., Chicago, IL, USA) program was used for analysis of data. Descriptive statistics were expressed with a mean \pm standard deviation. Continuous variables were assessed for normal distribution using the Shapiro-Wilk test. The data were found to be not normal. For the intergroup comparison of categorical data, the Pearson chi-square test was used, whereas for quantitative data, the Kruskal-Wallis test was used instead. For intragroup comparisons, Wilcoxon signed-rank test values were used. In addition, the analysis of BMD and $t$ values was carried out with the Statistic Calculator ${ }^{\circledR}$ (StatPac, Inc., Minneapolis, MN, USA) program. A $p<0.05$ value was considered significant in all tests.

\section{Results}

There were no significant differences between the groups in terms of demographic data and laboratory findings ( $p>0.05$, Table 2).

Radiographic Union Score for Hip scores were similar between groups I and II ( $p>0.05$, Table 2$)$. Fracture union occurred at the sixth month in all patients whose results were evaluated. The HHSs were found to be $70.2 \pm 8.3,72.8 \pm 8.1$, and $71.1 \pm 8.7$ in groups I, II, and III, respectively. This was regarded acceptable from a functioning perspective. No statistically significant difference was found between three groups ( $p>0.05$, Table 2).

There was also no difference between three groups with regard to hip and vertebrae BMD and $t$ scores ( $p>0.05$, Table 2 ). In addition, when $t$ and BMD scores before treatment were compared with those at 1 year after treatment, a benefit from ZolA treatment was observed in all three groups ( $p<0.05$, Table 3$)$.

\section{Adverse Effects}

During the analysis of data from this study, there were several data exclusions. In follow-up, results of four, six, and seven patients in groups I, II, and III, respectively, were excluded for the following reasons:
Table 2: Comparison of groups according to parameters

\begin{tabular}{lllll}
\hline Parameter & $\begin{array}{l}\text { Group I } \\
(n=26)\end{array}$ & $\begin{array}{l}\text { Group II } \\
(n=24)\end{array}$ & $\begin{array}{l}\text { Group III } \\
(n=23)\end{array}$ & p value \\
\hline Sex (F/M) & $13 / 13$ & $14 / 10$ & $12 / 11$ & $0.831^{*}$ \\
Side (R/L) & $15 / 11$ & $12 / 12$ & $13 / 10$ & $0.844^{*}$ \\
Anaesthesia & $20 / 6$ & $17 / 7$ & $18 / 5$ & $0.817^{*}$ \\
(general/regional) & & & & \\
ASA (2/3/4) & $16 / 8 / 2$ & $9 / 12 / 3$ & $6 / 12 / 5$ & $0.126^{*}$ \\
Age (year) & $70.8 \pm 5.5$ & $71.1 \pm 5.7$ & $74.8 \pm 6.9$ & $0.067^{* *}$ \\
BMI & $26.6 \pm 5.9$ & $27.6 \pm 6.1$ & $26.4 \pm 4.8$ & $0.680^{* *}$ \\
Albumin & $3.8 \pm 0.8$ & $3.7 \pm 0.7$ & $3.6 \pm 0.8$ & $0.669^{* *}$ \\
Hb & $11.7 \pm 1.7$ & $11.5 \pm 1.3$ & $11.3 \pm 1.6$ & $0.577^{* *}$ \\
CRP & $2.79 \pm 1.8$ & $3.69 \pm 3.0$ & $4.32 \pm 2.9$ & $0.112^{* *}$ \\
Creatinine & $1.21 \pm 0.4$ & $1.59 \pm 0.9$ & $1.91 \pm 1.3$ & $0.219^{* *}$ \\
Hospitalization & $7.5 \pm 3.6$ & $8.3 \pm 4.3$ & $8.2 \pm 4.3$ & $0.831^{* *}$ \\
HHS & $70.2 \pm 8.3$ & $72.8 \pm 8.1$ & $71.1 \pm 8.7$ & $0.573^{* *}$ \\
RUSH score & $22.5 \pm 2.6$ & $23.8 \pm 3.1$ & $22.8 \pm 2.3$ & $0.097^{* *}$ \\
\hline
\end{tabular}

${ }^{*}$ Chi-square test; ${ }^{* *}$ Kruskal-Wallis test; $\mathrm{BMI}$, body mass index

Table 3: In-group and intergroup comparison of $t$ score and bone mineral density according to the groups

\begin{tabular}{lllll}
\hline Parameter & $\begin{array}{l}\text { Group I } \\
(n=26)\end{array}$ & $\begin{array}{l}\text { Group II } \\
(n=24)\end{array}$ & $\begin{array}{l}\text { Group III } \\
(n=23)\end{array}$ & $p^{*}$ \\
\hline Vbmd & $0.76 \pm 0.1$ & $0.73 \pm 0.1$ & $0.71 \pm 0.1$ & 0.217 \\
Vbmd1 & $0.82 \pm 0.1$ & $0.79 \pm 0.1$ & $0.77 \pm 0.1$ & 0.294 \\
$p^{* *}$ & 0.035 & 0.043 & 0.047 & \\
Vt & $-2.79 \pm 1.1$ & $-3.10 \pm 0.8$ & $-3.13 \pm 0.7$ & 0.333 \\
Vt1 & $-1.99 \pm 1.6$ & $-2.42 \pm 1.3$ & $-2.52 \pm 1.2$ & 0.812 \\
$p^{* *}$ & 0.040 & 0.034 & 0.041 & \\
Fbmd & $0.72 \pm 1.0$ & $0.67 \pm 0.2$ & $0.65 \pm 0.1$ & 0.917 \\
Fbmd1 & $0.92 \pm 1.1$ & $0.84 \pm 0.3$ & $0.79 \pm 0.3$ & 0.806 \\
$p^{* *}$ & 0.049 & 0.025 & 0.039 & \\
Ft & $-2.42 \pm 0.9$ & $-2.52 \pm 0.4$ & $-2.59 \pm 0.6$ & 0.675 \\
Ft1 & $-1.85 \pm 1.1$ & $-2.24 \pm 0.5$ & $-2.25 \pm 0.5$ & 0.118 \\
$p^{* *}$ & 0.046 & 0.037 & 0.042 & \\
\hline
\end{tabular}

*Kruskal-Wallis test (intergroup); ${ }^{* *}$ paired $t$ test (in-group); Vbmd, vertebral bone mineral density; Vbmd1, 1-year follow-up, vertebral bone mineral density; $\mathrm{Vt}$, vertebral $\mathrm{L} 1-\mathrm{L} 4 t$ score; $\mathrm{Vt} 1$, one-year follow-up, vertebral L1-L4 $t$ score; Fbmd, femur bone mineral density; Fbmd1, 1-year follow-up, femur bone mineral density; Ft, femoral total $t$ score; Ft1, 1-year follow-up, femoral total $t$ score.

- Revision surgery. Three, one, and two patients underwent revision operations in groups I, II, and III, respectively.

- Death and lost to follow-up. Two, two, and three patients died and two, two, and three patients were lost to follow-up in groups I, II, and III, respectively. Therefore, in the final analyses, there were 26, 24, and 23 patients in groups I, II, and III, respectively. General adverse effects occurred in three patients in each of groups I and II and in two patients in group III. Finally, a delay in bone union occurred in three and two patients in groups I and II, respectively.

\section{Discussion}

Use of BPs in acute fractures has been debated with contradicting reports to beneficial or harmful effects in fracture healing. 
Controversial results are reported in animal studies, with delay in fracture healing, ${ }^{18-20}$ no effect, ${ }^{21,22}$ or even enhanced fracture healing ${ }^{23-27}$ as conclusions. It has been reported that BP treatment after hip fracture in elderly patients decreases mortality and that the timing of treatment does not have any negative effect on bone healing or incidence of complications. , 8,12 Health Outcomes and Reduced Incidence with Zoledronic Acid Once Yearly (HORIZON) trial is focussed on whether the early use of BPs after surgery (less than 3 months) would have any adverse or beneficial effects on fracture healing. Lyles et al. have reported that administration of annual ZolA infusion within 90 days of hip fracture operation is associated with decrease in the rate of new clinical fractures and increase in survival. ${ }^{7}$ Colón-Emeric et al. assessed HORIZON trials data, and they found that no association was observed between ZolA and delayed healing. ${ }^{9}$ In the meta-analysis and systematic review of $\mathrm{Li}$ et al., ${ }^{8}$ it was stated that early administration of BPs after surgery does not prolong bone healing process radiologically or clinically. Kim and colleagues performed osteosynthesis in 190 patients for intertrochanteric fractures. They divided the patients into three groups according to the start time of postoperative Risedronate treatment (1 week, 1 month, and 3 months). They found that there was no significant difference between the groups in terms of mean time to bone healing $(10.7,12.9$, and 12.3 weeks, respectively). They also reported that the 1-year functional results and the incidence of complications were similar. ${ }^{12}$

Our study shows that the timing of ZolA treatment does not affect fracture healing nor the incidence of complications in elderly patients with hip fractures. We also found administration of ZolA beneficial on both femoral and vertebral BMDs in all groups when compared with pretreatment values but with no significant differences between the groups. All fractures were observed to have healed. In addition, RUSH scores, used for objective evaluation of fractures, were found to be similar in all the groups $(p>0.05$; Table 2). These findings contrast to that of Kates et al. ${ }^{28}$ who have reported ZolA exerts an unfavourable effect on healing of bone. $\mathrm{Li}$ et al. also reported that BP use in the post-fracture period was associated with an increased probability of nonunion (odds ratio: 2.37, 95\% confidence interval: $1.13-4.96) .^{29}$

It has been reported in the literature that BP treatment started immediately after surgery is more effective in terms of antiresorptive effects and OP fracture prevention. Significant improvements in hip BMD and functional outcomes were reported with 12 months of BP treatment. ${ }^{8,30,31} \mathrm{Li}$ et al. ${ }^{30}$ divided 60 OP patients with intertrochanteric fractures to whom they treated surgically with the PFN. The treatment group was given an annual $5 \mathrm{mg}$ ZolA, calcium, and vitamin $D$, while the control group was given solely calcium and vitamin D. After 1 year, significant increases in BMD were established in both treatment and control groups. In the comparison between groups, it was stated that lumbar and hip BMD was significantly better in the ZolA group. However, in the 12th month after treatment, no significant differences were found between treatment and control groups with respect to HHSs. Five months after treatment, the fractures had healed in all patients. The mean duration of healing was found to be $13 \pm 3.2$ and $15 \pm 4.6$ weeks, respectively, for treatment and control groups. In the study by Cengiz et al. ${ }^{31}$ on 114 patients with intertrochanteric femoral fractures, no significant difference was found between treatment groups administered zoledronate and the placebo group in terms of American Society of Anesthesiologists (ASA) scores at 1 year after surgical treatment by osteosynthesis. The mean HHS was found to be 81.93 and 72.9 in treatment and control groups, respectively, being significantly better in the treatment group. The increase in BMD in the treatment group was found to be significantly higher at 1-year post-surgery. It was concluded that use of ZolA after surgery for intertrochanteric fractures helps to reduce mortality and to improve functionality in elderly patients.

In the present study, there was no significant difference between the three groups in terms of BMD and $t$ values (measured from the contralateral hip and vertebrae; $p>0.05$, Table 2). However, when $\mathrm{BMD}$ and $t$ scores were compared between the pretreatment period and at the first year, patients in all three groups were found to benefit from treatment ( $p<0.05$, Table 2). The HHSs were found to be $70.2 \pm 8.3,72.8 \pm 8.1$, and $71.1 \pm 8.7$ in groups I, II, and III, respectively, with functionally acceptable levels but no significant difference between groups ( $p>0.05$, Table 1 ).

It has been suggested that a delay in bone union should be evaluated at least 6 weeks after surgery and afterward. ${ }^{9}$ In similar studies, it was reported that these fractures healed in a mean of 10.7 to 12.3 weeks, and all fractures healed completely by the 24th week. ${ }^{12}$ However, there are important variations in the evaluation of fracture healing in orthopaedic trauma studies. ${ }^{32}$ When RUSH scores are used to evaluate union in femoral neck and intertrochanteric fractures, there was better agreement between radiologist and orthopaedist observers at the sixth months than at the third month. ${ }^{14}$ In the present study, and for these reasons, delay in union was evaluated at the third month and RUSH scores obtained at the sixth month. A delay in union was found in three cases in group I and two cases in group II. When evaluated with RUSH scores, where reliability and validity studies have been carried out, ${ }^{13,14,32}$ we found no significant difference between the groups.

There are many factors influencing mortality and duration of admission in patients undergoing operations for hip fractures. Two of these factors are haemoglobin and albumin values. ${ }^{33}$ In addition, in elderly patients with hip fractures, a high ASA value, advanced age, and male sex are associated with mortality. ${ }^{34}$ Zuradelli et al. ${ }^{35}$ reported that during treatment with ZolA, the incidence of hypocalcaemia and serum creatinine levels were found to be increased and that plasma calcium and creatinine levels should be monitored. In a study on women with osteoporosis over the age of 65 requiring long-term care, higher baseline C-reactive protein (CRP) and interleukin- 6 levels were found to be associated with worse physical performance and walking speed at 12 months independent of age, ZolA use, and comorbidity. ${ }^{36}$ In the present study, all of these factors (i.e., ASA, age, sex, albumin, haemoglobin, CRP, creatinine, and admission to hospital) known to be associated with morbidity and mortality in elderly patients with hip fractures were evaluated. No significant differences were found between the groups (Table 1).

Following the administration of ZolA, the possibility of moderate side effects such as myalgia, arthralgia, fever, or severe side effects such as avascular bone necrosis, cardiac arrhythmia, impairment of renal function, hypocalcaemia, delayed bone healing, and primary death have been noted. ${ }^{7}$ Lyles et al. ${ }^{7}$ reported that moderate side effects occurred at a higher rate in treated than placebo groups, but severe side effects were similar in both groups. In the study of Li et al., ${ }^{30}$ no important changes occurred in kidney and liver function tests in either group, but in the ZolA group, symptoms such as fever, influenza, and headache occurred in four patients and nausea or vomiting in two patients. In the present study, general adverse effects occurred in three patients each in groups I and II and in two patients in group III. 
Kim et al. ${ }^{12}$ stated that at the 24 th week after surgery, six patients had fixation failure and seven patients had died. In the study of Cengiz et al., ${ }^{31}$ the postoperative mortality rate was $14.3 \%(8 / 56)$ in the treatment group and $34.5 \%$ (20/58) in the control group, with a statistically significant difference between groups. In the present study, one, two, and two patients each underwent revision surgery, respectively, in groups I, II, and III. Mortality data showed one, one, and two patients had died in groups I, II, and III, respectively. The severity of osteoporosis affects the location of hip fracture; i.e., in patients with slight or marginal osteoporosis, intertrochanteric fractures occur more commonly, while in those with severe osteoporosis, the risk of femoral neck fracture increases. ${ }^{2}$ The fact that BMD values were higher in groups I and II than that in group III may be associated with this (Table 2).

\section{Strengths of the Study}

Factors such as race or environment may influence BMD values, ${ }^{17}$ and, in addition, the incidence of comorbid conditions may vary in different countries. Although there are various studies in the literature ${ }^{7,12,30,31}$ on this issue, to our knowledge, there is no previous study in our country regarding the effect of timing of ZolA treatment on bone healing. In addition, in the present study, bone healing was evaluated radiologically and clinically at specific periods. Therefore, in addition to its effect on bone healing, functional results and BMD values were also investigated.

\section{Limitations of the Study}

Although the required sample size was calculated before the study for the required power, the number of patients whose results were evaluated may be considered small. In addition, vitamin D deficiency is a commonly encountered problem in elderly patients with hip fractures and is associated with abnormal serum calcium levels. ${ }^{37}$ Individuals with low serum 25 -hydroxyvitamin D levels have an increased risk of hip fracture. ${ }^{38}$ Serum 25 -hydroxyvitamin $D$ levels may have been a confounding factor as these levels were not evaluated in the present study.

\section{Conclusion}

In elderly patients with hip fractures, the timing of ZolA treatment does not affect fracture healing or the incidence of complications. There are beneficial effects on both femoral and vertebral BMDs in all groups from treatment.

\section{References}

1. Tuzun S, Eskiyurt N, Akarirmak U, et al. Incidence of hip fracture and prevalence of osteoporosis in Turkey: the FRACTURK study. Osteoporos Int 2012;23(3):949-955. DOI: 10.1007/s00198-0111655-5.

2. Inan U, Tekcan A, Seber S, et al. Degree of osteoporosis affects the fracture site in older patients with proxımal femur fractures. Turkish Journal of Geriatrics/Türk Geriatri Dergisi 2011;14(3):204-207.

3. Bayrak A, Bayrak SK, Duramaz A, et al. Demographic and clinical results of proximal femoral fractures. Acta Medica Alanya 2017. 38.

4. Aslan A, Gülcü $A$, Özmeriç A. Outcomes of treatment in patients with elderly postmenopausal osteoporosis: comparision of oral and parenteral Bisphosphonates. Turk Osteoporoz Dergisi 2018;24(2):53. DOI: $10.4274 /$ tod.73645.

5. Eriksen EF, Lyles KW, Colón-Emeric CS, et al. Antifracture efficacy and reduction of mortality in relation to timing of the first dose of Zoledronic acid after hip fracture. J Bone Miner Res 2009;24(7): 1308-1313. DOI: 10.1359/jbmr.090209.
6. Turker M, Aslan A, Cirpar M, et al. Histological and biomechanical effects of zoledronate on fracture healing in an osteoporotic rat tibia model. Eklem Hastalik Cerrahisi 2016;27(1):9-15. DOI: 10.5606/ ehc.2016.03.

7. Lyles KW, Colón-Emeric CS, Magaziner JS, et al. Zoledronic acid and clinical fractures and mortality after hip fracture. N Engl J Med 2007;357(18):1799-1809. DOI: 10.1056/NEJMoa074941.

8. Li YT, Cai HF, Zhang ZL. Timing of the initiation of bisphosphonates after surgery for fracture healing: a systematic review and meta-analysis of randomized controlled trials. Osteoporos Int 2015;26(2):431-441. DOI: 10.1007/s00198-014-2903-2.

9. Colón-Emeric C, Nordsletten L, Olson S, et al. Association between timing of Zoledronic acid infusion and hip fracture healing. Osteoporos Int 2011;22(8):2329-2336. DOI: 10.1007/s00198-010-14731.

10. Einhorn TA. The science of fracture healing. J Orthop Trauma 2005;19(Suppl. 10):S4-S6. DOI: 10.1097/00005131-200511101-00002.

11. Gerstenfeld LC, Sacks DJ, Pelis M, et al. Comparison of effects of the Bisphosphonate alendronate versus the RANKL inhibitor denosumab on murine fracture healing. J Bone Miner Res 2009;24(2):196-208. DOI: $10.1359 /$ jbmr.081113.

12. Kim T, Ha Y, Kang B, et al. Does early administration of bisphosphonate affect fracture healing in patients with intertrochanteric fractures? J Bone Joint Surg Br 2012;94(7):956-960. DOI: 10.1302/0301620X.94B7.29079.

13. Bhandari $\mathrm{M}, \mathrm{Chiavaras} \mathrm{M}$, Ayeni $\mathrm{O}$, et al. Assessment of radiographic fracture healing in patients with operatively treated femoral neck fractures. J Orthop Trauma 2013;27(9):e213-e219. DOI: 10.1097/ BOT.0b013e318282e692.

14. Bhandari M, Chiavaras MM, Parasu N, et al. Radiographic union score for hip substantially improves agreement between surgeons and radiologists. BMC Musculoskelet Disord 2013;14(1):70. DOI: 10.1186/1471-2474-14-70.

15. Nilsdotter A, Bremander A. Measures of hip function and symptoms: harris hip score (HHS), hip disability and osteoarthritis outcome score (HOOS), oxford hip score (OHS), lequesne index of severity for osteoarthritis of the hip (LISOH), and american academy of orthopedic surgeons (AAOS) hip and knee questionnaire. Arthritis Care Res (Hoboken) 2011;63(Suppl 11):S200-S207. DOI: 10.1002/ acr.20549.

16. Tang P, Hu F, Shen J, et al. Proximal femoral nail antirotation versus hemiarthroplasty: a study for the treatment of intertrochanteric fractures. Injury 2012;43(6):876-881. DOI: 10.1016/j.injury.2011.11.008.

17. Aslan A, Karakoyun O, Güler E, et al. Evaluation of bone mineral density, osteoporosis prevalence and regional risk factors in Turkish women living in Kastamonu: KASTÜRKOS study. Eklem hastaliklari ve cerrahisi= Joint diseases \& related surgery 2012;23(2):62-67.

18. Matos MA, Tannuri U, Guarniero R. The effect of zoledronate during bone healing. J Orthop Traumatol 2010;11(1):7-12. DOI: 10.1007/ s10195-010-0083-1.

19. Li J, Mori S, Kaji Y, et al. Effect of bisphosphonate (incadronate) on fracture healing of long bones in rats. J Bone Miner Res 1999;14(6):969-979. DOI: 10.1359/jbmr.1999.14.6.969.

20. Li C, Mori S, Li J, et al. Long-term effect of incadronate disodium (YM-175) on fracture healing of femoral shaft in growing rats. J Bone Miner Res 2001;16(3):429-436. DOI: 10.1359/jbmr.2001.16.3.429.

21. Bauss F, Schenk RK, Hort S, et al. New model for simulation of fracture repair in full-grown beagle dogs: model characterization and results from a long-term study with ibandronate. J Pharmacol Toxicol Methods 2004;50(1):25-34. DOI: 10.1016/j.vascn.2003.11.003.

22. Munns CF, Rauch F, Zeitlin L, et al. Delayed osteotomy but not fracture healing in pediatric osteogenesis imperfecta patients receiving pamidronate. J Bone Miner Res 2004;19(11):1779-1786. DOI: 10.1359/ JBMR.040814.

23. Greiner SH, Wildemann B, Back DA, et al. Local application of Zoledronic acid incorporated in a poly(D,L-lactide)-coated implant accelerates fracture healing in rats. Acta Orthop 2008;79(5):717-725. DOI: 10.1080/17453670810016768. 
24. Rozental TD, Vazquez MA, Chacko AT, et al. Comparison of radiographic fracture healing in the distal radius for patients on and off bisphosphonate therapy. J Hand Surg Am 2009;34(4):595-602. DOI: 10.1016/j.jhsa.2008.12.011.

25. Amanat $N$, Brown R, Bilston LE, et al. A single systemic dose of pamidronate improves bone mineral content and accelerates restoration of strength in a rat model of fracture repair. J Orthop Res 2005;23(5):1029-1034. DOI: 10.1016/j.orthres.2005.03.004.

26. Little DG, McDonald M, Bransford R, et al. Manipulation of the anabolic and catabolic responses with OP-1 and Zoledronic acid in a rat critical defect model. J Bone Miner Res 2005;20(11):2044-2052. DOI: 10.1359/JBMR.050712.

27. Bransford R, Goergens E, Briody J, et al. Effect of Zoledronic acid in an L6-L7 rabbit spine fusion model. Eur Spine J 2007;16(4):557-562. DOI: 10.1007/s00586-006-0212-y.

28. Kates SL, Ackert-Bicknell CL. How do bisphosphonates affect fracture healing? Injury 2016;47:S65-S68. DOI: 10.1016/S0020-1383(16) 30015-8.

29. Li C, Wang H-R, Li X-L, et al. The relation between Zoledronic acid infusion and interbody fusion in patients undergoing transforaminal lumbar interbody fusion surgery. Acta Neurochir (Wien) 2012;154(4):731-738. DOI: 10.1007/s00701-012-1283-7.

30. Li Y, Zhao WB, Wang DL, et al. Treatment of osteoporotic intertrochanteric fractures by Zoledronic acid injection combined with proximal femoral nail anti-rotation. Chin J Traumatol 2016;19(5):259-263. DOI: 10.1016/j.cjtee.2016.07.001.

31. Cengiz Ö, Polat G, Karademir G, et al. Effects of zoledronate on mortality and morbidity after surgical treatment of hip fractures. Adv Orthop 2016;2016:3703482. DOI: 10.1155/2016/ 3703482.

32. Maiettini D, Bisaccia M, Caraffa $A$, et al. Feasibility and value of radiographic union score hip fracture after treatment with intramedullary nail of stable hip fractures. Acta Inform Med 2016;24(6):394-396. DOI: 10.5455/aim.2016.24.394-396.

33. Güler A, Doğukan $M$, Kaya R, et al. Retrospective evaluation of the effects of albumin and hemoglobin values on the duration of hospital stay and mortality in elderly patients operated for hip fracture. Adıyaman Üniversitesi Sağlık Bilimleri Dergisi 2018;4(1):637-647.

34. Atay T, Ceylan BG, Özmeriç A, et al. The effects of related factors on one-and two-year mortality after a hip fracture in elderly Turkish patients. Balkan Med J 2010;27(2):127-131.

35. Zuradelli M, Masci G, Biancofiore G, et al. High incidence of hypocalcemia and serum creatinine increase in patients with bone metastases treated with Zoledronic acid. Oncologist 2009;14(5): 548-556. DOI: 10.1634/theoncologist.2008-0227.

36. Langmann GA, Perera S, Ferchak MA, et al. Inflammatory markers and frailty in long-term care residents. J Am Geriatr Soc 2017;65(8): 1777-1783. DOI: 10.1111/jgs.14876.

37. Pieper CF, Colon-Emetic C, Caminis J, et al. Distribution and correlates of serum 25 -hydroxyvitamin D levels in a sample of patients with hip fracture. Am J Geriatr Pharmacother 2007;5(4):335-340. DOI: 10.1016/j. amjopharm.2007.12.004.

38. Lv QB, Gao X, Liu X, et al. The serum 25-hydroxyvitamin D levels and hip fracture risk: a meta-analysis of prospective cohort studies. Oncotarget 2017;8(24):39849-39858. DOI: 10.18632/oncotarget. 16337. 